\title{
Aliveness: Perceived Instability from a Passive Haptic Texture Rendering System
}

\author{
Seungmoon Choi and Hong Z. Tan \\ Haptic Interface Research Laboratory \\ Purdue University \\ 1285 EE Building, West Lafayette, IN 47907-1285 \\ http://www.ece.purdue.edu/HIRL \\ $\{$ chois, hongtan\}@purdue.edu
}

\begin{abstract}
This paper reports new findings of our ongoing research on perceived instability that human users frequently experience from interacting with virtual textures rendered with a force-feedback haptic interface. Our work is aimed at a better understanding of a new type of perceived instability called "aliveness", which was discovered during our previous psychophysical experiments performed using a popular texture rendering method (spring model with fixed force directions) and a common texture model (positionbased sinusoidal grating). We first examine the perceptual and physical characteristics of the proximal stimuli that cause the perception of aliveness in virtual textures. It leads to the hypothesis that the virtual environment model used for computing texture-perturbing forces, not the traditional control-related instabilities of the haptic texture rendering system, is responsible for aliveness perception. We show that this conjecture is true by applying passivity-based stability theory to the position and force data measured during user interaction with virtual textures. Examples of the data where the haptic texture rendering system is passive (therefore stable) and aliveness is perceived are provided to substantiate our conclusions. Our results point to the importance of designing haptic texture rendering methods and models that are free of perceptual artifacts.
\end{abstract}

\section{INTRODUCTION}

This paper reports new findings of our ongoing research on the perceived instability that human users frequently experience from virtual textures rendered with a forcefeedback haptic interface (see [3][4][5] for a series of our previous studies). By perceived instability, we refer to all unrealistic sensations (such as buzzing or apparent aliveness of a surface) that cannot be attributed to the physical properties of a textured surface rendered with a force-feedback device. Insufficient understanding of the conditions under which the textured virtual objects are free of such perceptual artifacts may significantly undermine the usefulness of haptic texture rendering techniques to virtual reality applications and the validity of psychophysical experiments using haptic virtual textures.

In general, there are two main sources of perceived instability in haptic rendering: unstable control of the haptic interface and improper environment model (texture rendering method and model, as in our case). Both issues have been extensively studied for the virtual wall problem where the goal is to haptically render a stiff wall under stable control of the haptic interface. The environment dynamics model of the virtual wall is typically composed of a mechanical spring and a damper. For this benchmark, studies on the stable control of the haptic interface have been widely undertaken (see [1][8][13] for recent ones), as well as those on the perceptual effects of the model parameters on the perceived hardness (for examples, see [16][11]). These studies have led to the general consensus that the simple spring and damper model can effectively deliver the target percept of flat-wall hardness without perceived instability, provided that the parameters are carefully chosen.

Whereas the rendering of a virtual wall/surface provides the macro-geometry of a virtual object, haptic textures supply the micro-geometry of a virtual object. Most research on haptic texture rendering has focused on the development of efficient computational algorithms (see [7][9][12][14][15][17][6]; see also [3] for a review). Our previous studies [3][4][5] were among the first to investigate perceived instability during haptic texture rendering and the sources of this perceptual phenomenon. Our first study quantified the level of perceived stability/instability during haptic texture rendering [3]. We conducted psychophysical experiments in which subjects interacted with a textured surface rendered with a PHANToM force-feedback haptic interface (SensAble Technologies; Woburn, MA) and judged the maximum stiffness of the surface that could be rendered without the perception of instability. We found that the useful parameter space for stable texture rendering was very small. The stiffest textured surfaces rendered within the stable parameter space feel like soft corduroy, thereby greatly limiting the range of textures that can be rendered without perceptual artifacts.

Our next study investigated the characteristics of proximal stimuli (position, force and acceleration at or near the tip of the PHANToM stylus) that invoked the perception of instability [4]. We observed that whenever the 


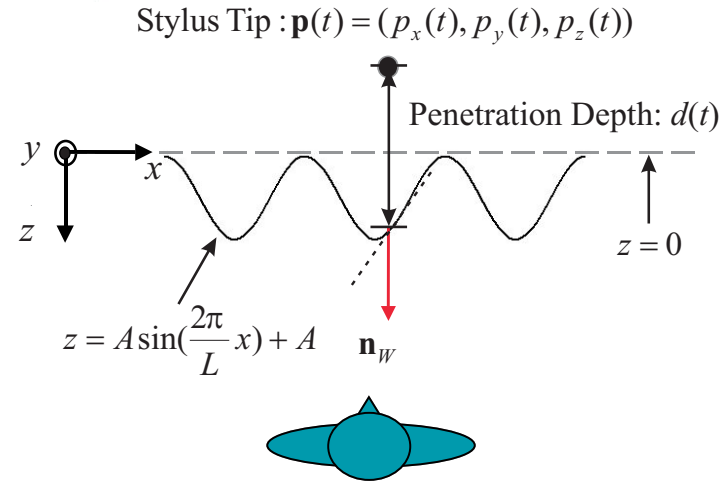

Fig. 1. Illustration of parameters used in texture rendering.

subject perceived "buzzing" from the textured surfaces and subsequently declared the textures to be unrealistic and unstable, a high-frequency noise was present in measured data. The frequency range of this buzzing noise was found to be consistent with that of the mechanical resonance of the PHANToM $(192-242 \mathrm{~Hz})$. These results implies that studies on the stable control of haptic interfaces for virtual wall rendering need to be extended to haptic texture rendering [3][4].

Our most recent study compared the effects of different collision detection algorithms on the perceived instability of haptic texture rendering [5]. It revealed another kind of perceived instability, often described as "aliveness" by subjects, that was perceptually distinct from traditional control instabilities such as chattering and buzzing.

In the current study, we investigate the role played by haptic interface controller and the environment dynamics model on the perception of aliveness. In particular, we try to answer the question of whether the texture rendering system can be stable when users feel aliveness. To assess the stability of the texture rendering system, passivitybased stability theory is applied to data gathered from user interactions with virtual textured surfaces. If aliveness perception persists under conditions where the texture rendering system is deemed passive, then we can conclude that aliveness perception is due to the improper model and rendering algorithm of the textured surfaces.

\section{PERCEIVED INSTABILITY: ALIVENESS}

This section describes the conditions under which aliveness is perceived and examines the perceptual and physical characteristics of aliveness through the analysis of measured data.

The texture model used in our previous study that revealed the aliveness percept [5] consists of onedimensional sinusoidal gratings. The heights of these gratings are represented by $z=A \sin \left(\frac{2 \pi}{L} x\right)+A$ in the PHANToM world coordinate frame (see Fig. 1). Using this model, the penetration depth $d(t)$ is defined as

$$
d(t)=\left\{\begin{array}{cl}
0 & \text { if } p_{z}(t)>h\left(p_{x}(t)\right) \\
A \sin \left(\frac{2 \pi}{L} p_{x}(t)\right)+A-p_{z}(t) & \text { if } p_{z}(t) \leq h\left(p_{x}(t)\right)
\end{array}\right.
$$

where $\mathbf{p}(t)=\left(p_{x}(t), p_{y}(t), p_{z}(t)\right)$ is the position of the PHANToM stylus tip, and $h\left(p_{x}(t)\right)=A \sin \left(\frac{2 \pi}{L} p_{x}(t)\right)+A$ is the height of the texture model at $p_{x}(t)$. The rendering force is computed as $\mathbf{F}_{\text {mag }}(t)=K d(t) \mathbf{n}_{W}$, where $K$ denotes stiffness and $\mathbf{n}_{W}$ denotes the normal of the underlying plane. This method, which was proposed in [12], perturbs only force magnitudes according to the texture model. It has been used in psychophysical studies using virtual textures (for example, see [18]). Perception of aliveness occurred with surfaces rendered with the aforementioned texture rendering method and model for two exploration modes tested: free exploration and stroking. During free exploration, subjects were free to interact with the textured surfaces in any manner. During stroking, subjects were instructed to move the PHANToM stylus laterally across the sinusoidal gratings (i.e., along the $x$-axis in Fig. 1).

Our previous work found that perceived aliveness during free exploration is due to a perceptible change in force while the stylus is perceived to be stationary in space [5]. Aliveness sensation was usually reported when the subject positioned the stylus inside the textured surface. Fig. 2(a) demonstrates this finding with position and force data measured during free exploration of the textured surface (rendered with a stiffness value one standard deviation above the stiffness threshold for perceptually stable texture rendering). In this figure, force along the cylindrical axis of the stylus, $F_{z}^{S}(t)$, is plotted with respect to the displacement of the stylus, $\left(p_{x}(t), p_{z}(t)\right)$, for a short period of time $(400 \mathrm{~ms})$. Note that $F_{z}^{S}(t)$ is not plotted against $p_{y}(t)$ since our texture model does not vary along the $y$-axis. Also shown are three projections. The projection on the $p_{x}(t)-p_{z}(t)$ plane shows that the tip of the stylus moved by less than $0.56 \mathrm{~mm}$ in the $x$-direction and $0.94 \mathrm{~mm}$ in the $z$-direction. This movement magnitude is probably below the human detection threshold when the hand is moving in free space. The corresponding change in force magnitude, however, was large enough to be perceptible $\left(\max F_{z}^{S}(t)-\min F_{z}^{S}(t)=0.59 \mathrm{~N}\right)$. Fig. 2(b) shows the data collected during stroking when the subject perceived aliveness. In this figure, we can observe many "ripples" with force magnitudes on the order of $0.5 \mathrm{~N}$ despite a very short time period $(400 \mathrm{~ms})$. This seems to be consistent with the subjects' reports that they felt "pulsating" textured surfaces during stroking.

A particularly interesting finding is that the perceptual and physical characteristics of aliveness are quite different from those of buzzing - a typical perceived instability reported in the literature on the hard wall rendering problem and in our previous work. The difference between aliveness and buzzing can be clearly observed in the 


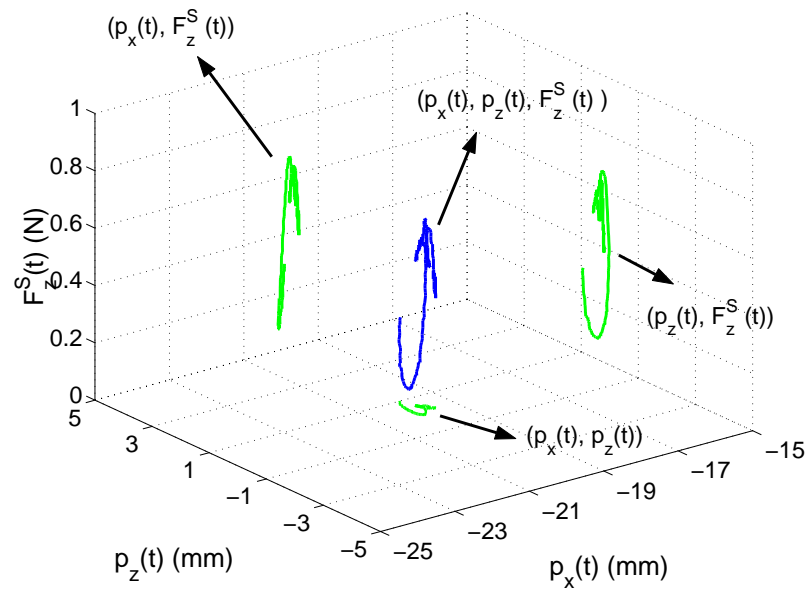

(a) Free exploration and $(A, L, K)=(1 \mathrm{~mm}, 1 \mathrm{~mm}, 0.4 \mathrm{~N} / \mathrm{mm})$.

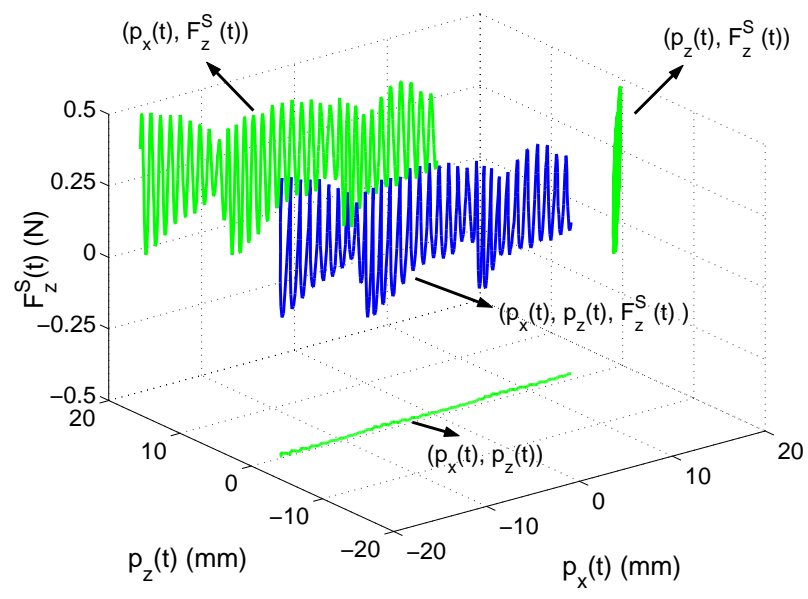

(b) Stroking and $(A, L, K)=(1 \mathrm{~mm}, 1 \mathrm{~mm}, 0.5 \mathrm{~N} / \mathrm{mm})$.

Fig. 2. Characteristics of aliveness.

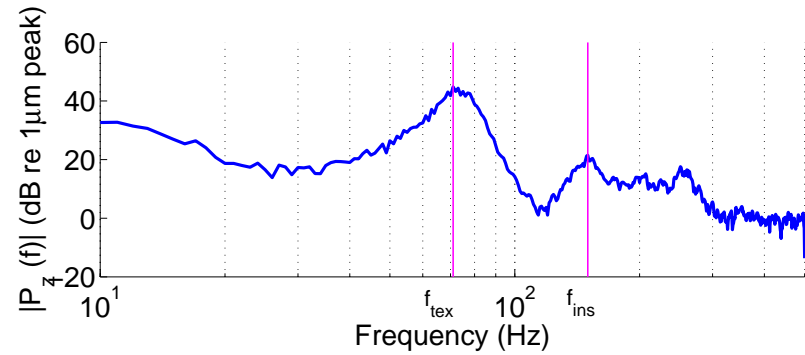

(a) Stroking and $(A, L, K)=(1 \mathrm{~mm}, 2 \mathrm{~mm}, 1.2 \mathrm{~N} / \mathrm{mm})$.

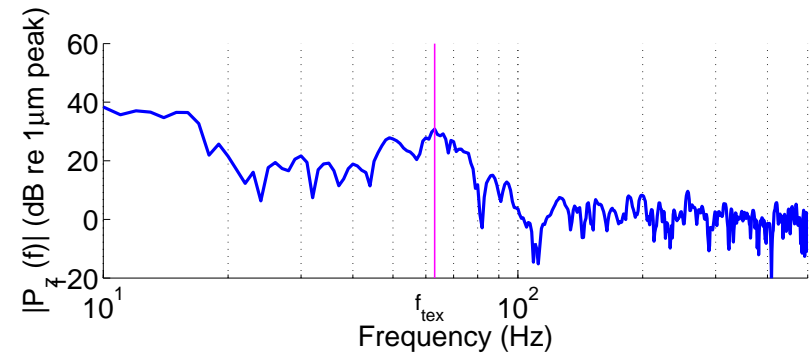

(b) Stroking and $(A, L, K)=(1 \mathrm{~mm}, 1 \mathrm{~mm}, 0.5 \mathrm{~N} / \mathrm{mm})$.

Fig. 3. Power spectral densities of $p_{z}(t)$.

frequency spectrum of position data measured along the $\mathrm{z}-$ axis (i.e., perpendicular to the wall underlying the textured surface). Fig. 3(a) shows the case where buzzing was apparent in the virtual textures rendered with stiffness values much higher than the threshold for perceptually stable rendering. In this figure, two spectral peaks are present: one for texture information at around $f_{\text {tex }}=71$ $\mathrm{Hz}$ and the other for instability perception at around $f_{\text {ins }}=150 \mathrm{~Hz}$. This indicates that the subject perceived the texture information corrupted by the high frequency noise that is responsible for the perception of buzzing. As the stiffness gain was decreased to be close to the threshold for perceptually stable texture rendering, buzzing was reduced and aliveness became the primary criterion used by the subject to declare unstable textures. Fig. 3(b) shows the typical data for aliveness. It is apparent that aside from the spectral peak for texture perception $\left(f_{\text {tex }}=63 \mathrm{~Hz}\right)$, no other distinctive spectral components can be observed. These data strongly suggest that aliveness may be inherently different from buzzing, the traditional control instability for haptic rendering.

\section{PASSIVITY OF RENDERING}

A question that naturally follows from prior observations is what is responsible for the perception of aliveness. Since the perceptual and physical characteristics of aliveness are qualitatively different from those of controlrelated instability and the environment dynamics for texture rendering is designed to create temporal vibrations unlike that for virtual wall rendering, we suspected that aliveness may be caused by the virtual environment dynamics $\left(\mathbf{F}_{\text {mag }}(t)\right.$ and the sinusoidal grating texture model) rather than control instability.

To investigate this hypothesis, we examined whether it is possible for a human user to perceive aliveness while the texture rendering system including the forcefeedback device is stable in the control sense. For this purpose, we applied passivity-based stability theory on the data measured from a user interacting with virtual textured surfaces. Since passivity is a sufficient condition for stability [8], our hypothesis can be confirmed if we 
Haptic Texture Rendering System

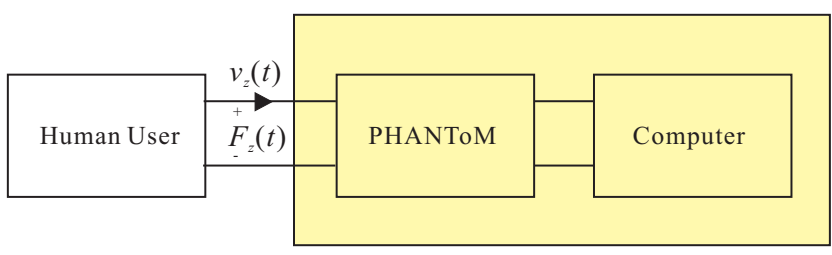

Fig. 4. Effort and flow of haptic texture rendering system.

find cases in which a human user feels aliveness from a passive texture rendering system.

In order to judge the passivity of the texture rendering system, we used a passivity observer (PO) [8]. The PO is an on-line observer for monitoring the energy flow of a dynamic system. The system is passive if the PO is positive for all time indices under consideration [8]. For the texture rendering system shown in Fig. 4, the PO with zero initial energy storage is defined as

$$
P O(k)=\sum_{i=1}^{k} F_{z}^{W}(i \Delta t) v_{z}(i \Delta t) \Delta t,
$$

where $\Delta t$ is the sampling time, $k$ is the time index for samples, $F_{z}^{W}(t)$ is the measured force at the stylus of the PHANToM along the $z$-axis of the PHANToM world coordinate frame, and $v_{z}(t)$ is the velocity of the stylus along the $z$-axis. We only considered $F_{z}^{W}(t)$ because the PHANToM only applies forces in the $z$-direction when $\mathbf{F}_{\text {mag }}(t)$ is used.

The measurement data used by the PO were collected under a variety of experimental conditions varying in subject (one male (S1) and one female (S2)), stiffness value, and exploration mode (free exploration and stroking). The two subjects had both participated in our previous experiments in which the thresholds for perceptually stable texture rendering were measured [5]. The parameters for sinusoidal gratings were $A=1 \mathrm{~mm}$ and $L=2 \mathrm{~mm} . F_{z}^{W}(t)$ was measured with a $3 \mathrm{D}$ force/torque sensor mounted on the last link of the PHANToM that was closest to the stylus (see [4] for instrumentation details). Due to the poor resolution of velocity estimates derived directly from the PHANToM position encoders [2], $v_{z}(t)$ was estimated using the end-fit first-order adaptive windowing technique [10].

\section{EXPERIMENTAL RESULTS}

Four representative data plots are shown in this section for the cases where (a) the haptic texture rendering system is stable in both the control and perceptual sense, (b) rendering is passive but aliveness is perceived (free exploration), (c) same as (b) with stroking, and (d) rendering is active and aliveness is perceived.

Fig. 5(a) shows the results of one experimental condition under which subject S2 could maintain stationary contact between the stylus of the PHANToM and the virtual textured surface without feeling any instability. The stiffness, $K=0.2 \mathrm{~N} / \mathrm{mm}$, was about one standard deviation below the threshold for perceptually stable texture rendering under the same condition $(A=1 \mathrm{~mm}$ and $L=2$ $\mathrm{mm})$. The top panel shows $p_{z}(t)$ over ten seconds, and the middle panel shows $F_{z}^{W}(t)$. Both measured variables rarely exhibit abrupt changes. As expected, the PO remains positive at all time.

The next figure, Fig. 5(b), is for an experimental condition measured with $K=0.5 \mathrm{~N} / \mathrm{mm}$, which is about one standard deviation above the threshold for stable rendering. Subject $\mathrm{S} 1$ reported that the texture felt alive in this condition. Consistent with the subject's perception, much larger fluctuations are observed in both the $p_{z}(t)$ and $F_{z}^{W}(t)$ plots. However, the PO remains to be positive indicating that perceived instability can occur even when the texture rendering system is passive and stable.

A similar case was also found for stroking. The data shown in Fig. 5(c) were measured when subject S1 perceived apparent aliveness but no high-frequency buzzing noises. The stiffness, $K=0.8 \mathrm{~N} / \mathrm{mm}$, was one standard deviation above the corresponding threshold for stable texture rendering. The top panel shows the position data along the lateral direction, $p_{x}(t)$, for stroking motion. The next two panels $\left(p_{z}(t)\right.$ and $F_{z}^{W}(t)$, respectively) show the abrupt changes in proximal stimuli that resulted in the perception of aliveness. In particular, the magnitude of force variations is up to about $2 \mathrm{~N}$. Despite the apparent aliveness, however, the PO (the bottom panel) remains positive, and therefore the texture rendering system is passive and stable.

The last plot, Fig. 5(d), shows an example where the texture rendering system is active during stroking. The data were measured when subject S2 stroked the textured surface rendered with very high stiffness $(K=$ $1.2 \mathrm{~N} / \mathrm{mm})$. The subject reported that both aliveness and high-frequency buzzing noises were present under this experimental condition. Aliveness can be observed in the plots of $p_{z}(t)$ and $F_{z}^{W}(t)$ in terms of very small positional variations but relatively large force variations. High-frequency buzzing shows up as the spectral peak at $150 \mathrm{~Hz}$ in the power spectrum of $p_{z}(t)$ that was shown earlier in Fig. 3(a). In the bottom panel of Fig. 5(d), the PO (the bottom panel) turns negative, indicating that the texture rendering system was active.

\section{SUMMARY}

We have shown in this paper that some currently widely used texture rendering methods and models may introduce artifacts that result in unrealistic sensations. We first went over the perceptual and physical characteristics of perceived instability of aliveness using data measured from a user interacting with a virtual textured wall. This 

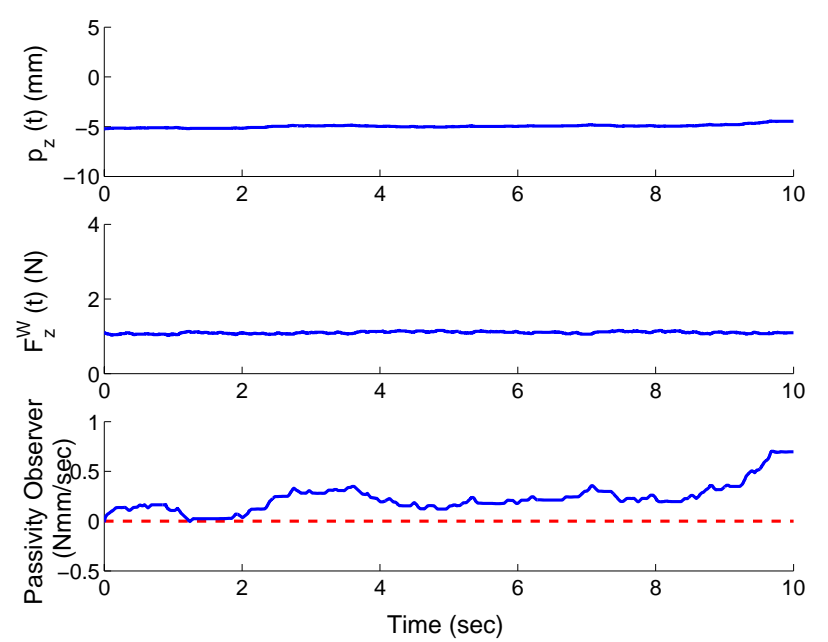

(a) Passive rendering during free exploration without instability perception (Subject S2, $A=1 \mathrm{~mm}, L=2 \mathrm{~mm}$, and $K=0.2 \mathrm{~N} / \mathrm{mm}$ ).

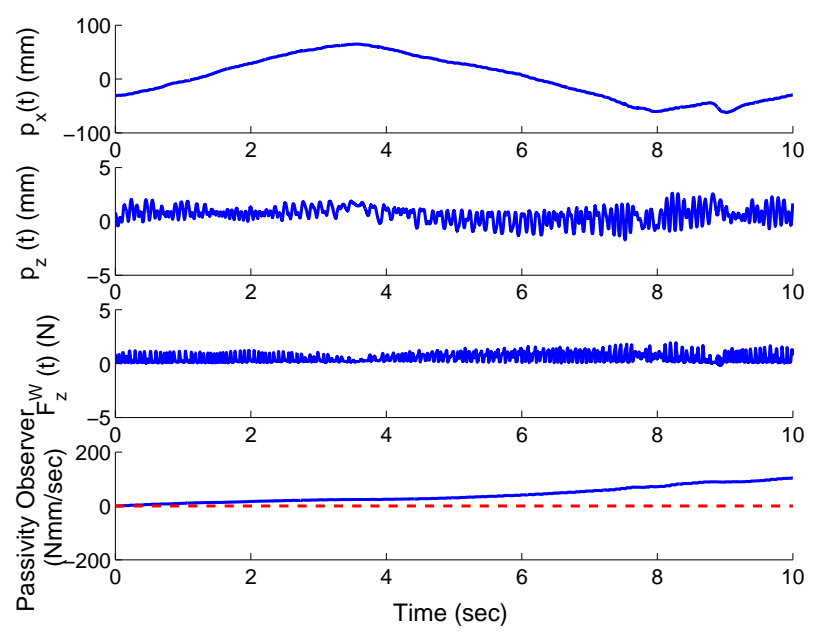

(c) Passive rendering during stroking with aliveness perception (Subject S1, $A=1 \mathrm{~mm}, L=2 \mathrm{~mm}$, and $K=0.8 \mathrm{~N} / \mathrm{mm}$ ).
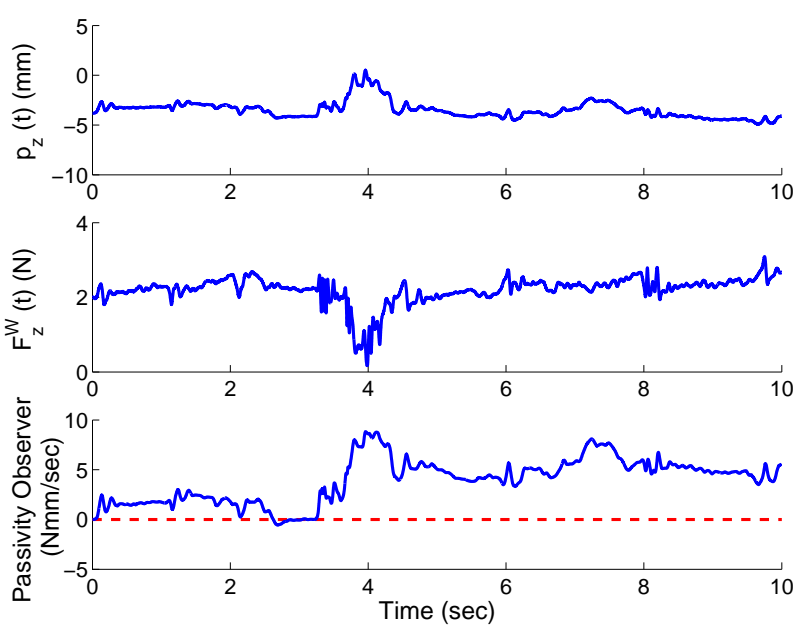

(b) Passive rendering during free exploration with aliveness perception (Subject S1, $A=1 \mathrm{~mm}, L=2 \mathrm{~mm}$, and $K=0.5 \mathrm{~N} / \mathrm{mm}$ ).

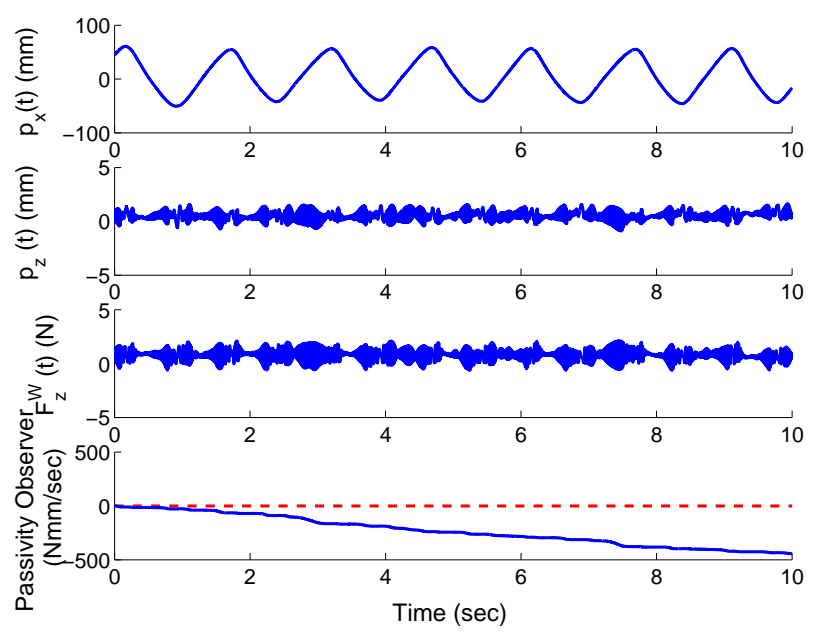

(d) Active rendering during stroking with aliveness and buzzing perception (Subject S2, $A=1 \mathrm{~mm}, L=2 \mathrm{~mm}$, and $K=1.2 \mathrm{~N} / \mathrm{mm}$ ).

Fig. 5. Experimental results.

analysis led to the hypothesis that the unstable control of a force-feedback haptic interface may not be the source of aliveness perception. By applying passivity-based control theory on the experimentally collected data, we showed that the conjecture is true and that the texture rendering method and model are responsible for aliveness perception.

Our previous study found that perceived instability such as buzzing is due to unstable control of the PHANToM haptic interface. Our current study suggests that, unlike virtual wall rendering, texture rendering methods and models can invoke the perception of unrealistic sensations such as aliveness while the haptic interface is stably controlled. When combined, these findings suggest that the effects of both device control and environment dynamics should be taken into account to accomplish perceptually realistic rendering of haptic textures. This requirement brings up two new research issues. How do we design texture rendering methods and models that do not induce perceptual artifacts? How do we extend the stable control techniques for virtual wall rendering to texture rendering? We shall pursue these questions in the future.

\section{ACKNOWLEDGEMENTS}

This work was supported in part by a National Science Foundation (NSF) Faculty Early Career Development (CAREER) Award under Grant 9984991-IIS and in part by an NSF award under Grant 0098443-IIS. The authors 
wish to thank Prof. Blake Hannaford and Dr. Jee-Hwan Ryu for the discussions on the passivity observer, and Prof. Vincent Hayward for the help on velocity estimation.

\section{REFERENCES}

[1] R. J. Adams and B. Hannaford, "Stable haptic interaction with virtual environments," IEEE Transactions on Robotics and Automation, vol. 15, no. 3, pp. 465-474, June 1999.

[2] M. C. Çavuşoğlu, D. Feygin, and F. Tendick, "A critical study of the mechanical and electrical properties of the PHANToM ${ }^{T M}$ haptic interface and improvements for high performance control," Presence, vol. 11, no. 6, pp. 555$568,2002$.

[3] S. Choi and H. Z. Tan, "An analysis of perceptual instability during haptic texture rendering," in Proceedings of the 10th International Symposium on Haptic Interfaces for Virtual Environment and Teleoperator Systems (IEEE VR 2002), 2002, pp. 129-136.

[4] S. Choi and H. Z. Tan, "A study on the sources of perceptual instability during haptic texture rendering," in Proceedings of the IEEE International Conference on Robotics and Automation, 2002, pp. 1261-1268.

[5] S. Choi and H. Z. Tan, "An experimental study of perceived instability during haptic texture rendering: Effects of collision detection algorithm," in Proceedings of the 11th International Symposium on Haptic Interfaces for Virtual Environment and Teleoperator Systems (IEEE VR 2003), 2003, pp. 197-204.

[6] M. A. Costa and M. R. Cutkosky, "Roughness perception of haptically displayed fractal surfaces," in Proceedings of the ASME Dynamic Systems and Control Division, vol. 692, 2000, pp. 1073-1079.

[7] J. P. Fritz and K. E. Barner, "Stochastic models for haptic texture," in Proceedings of SPIE's International Symposium on Intelligent Systems and Advanced Manufacturing Telemanipulator and Telepresence Technologies III, 1996, pp. 34-44.
[8] B. Hannaford and J.-H. Ryu, "Time-domain passivity control of haptic interfaces," IEEE Transactions on Robotics and Automation, vol. 18, no. 1, pp. 1-10, 2002.

[9] C. Ho, C. Basdogan, and M. A. Srinivasan, "Efficient pointbased rendering techniques for haptic display of virtual objects," Presence, vol. 8, no. 5, pp. 477-491, 1999.

[10] F. Janabi-Sharifi, V. Hayward, and C.-S. J. Chen, "Discretetime adaptive windowing for velocity estimation," IEEE Transactions on Control System Technology, vol. 8, no. 6, pp. 1003-1009, 2000.

[11] D. A. Lawrence, L. Y. Pao, A. M. Dougherty, M. A. Salada, and Y. Pavlou, "Rate-hardness: A new performance metric for haptic interfaces," IEEE Transactions on Robotics and Automation, vol. 16, no. 4, pp. 357-371, 2000.

[12] T. H. Massie, "Initial haptic explorations with the phantom: Virtual touch through point interaction," Master's thesis, MIT, Feb. 1996.

[13] B. E. Miller, E. Colgate, and R. A. Freeman, "Guaranteed stability of haptic systems with nonlinear virtual environments," IEEE Transactions on Robotics and Automation, vol. 16, no. 6, pp. 712-719, 2000.

[14] M. Minsky and S. J. Lederman, "Simulated haptic textures: Roughness," in Proceedings of the ASME Dynamic Systems and Control Division, vol. 58. ASME, 1996, pp. 421-426.

[15] A. M. Okamura, J. T. Dennerlein, and R. D. Howe, "Vibration feedback models for virtual environments," in Proceedings of the IEEE International Conference on Robotics and Automation, 1998, pp. 674-679.

[16] L. B. Rosenberg and B. D. Adelstein, "Perceptual decomposition of virtual haptic surfaces," in Proceedings of IEEE Symposium on Research Frontiers in Virtual Reality, 1993, pp. 46-53.

[17] J. Siira and D. K. Pai, "Haptic texturing - a stochastic approach," in Proceedings of the IEEE International Conference on Robotics and Automation, 1996, pp. 557-562.

[18] J. M. Weisenberger, M. J. Krier, and M. A. Rinker, "Judging the orientation of sinusoidal and square-wave virtual gratings presented via $2-\mathrm{DOF}$ and 3-DOF haptic interfaces," Haptics-e (http://www.haptics-e.org), vol. 1, no. 4, 2000. 